\title{
Determination of transverse relaxation rates in systems with scalar-coupled spins: The role of antiphase coherences
}

\author{
Takuya F. Segawa ${ }^{\mathrm{a}, 1}$, Geoffrey Bodenhausen ${ }^{\mathrm{a}, \mathrm{b}, \mathrm{c}, \mathrm{d}, *}$ \\ a Institut des Sciences et Ingénierie Chimiques, Ecole Polytechnique Fédérale de Lausanne (EPFL), Batochime, 1015 Lausanne, Switzerland \\ ${ }^{\mathrm{b}}$ Département de Chimie, Ecole Normale Supérieure, 24 Rue Lhomond, 75231 Paris Cedex 05, France \\ ' Université Pierre-et-Marie Curie, Paris, France \\ ${ }^{\mathrm{d}}$ UMR 7203 CNRS, Paris, France
}

\section{A R T I C L E I N F O}

\section{Article history:}

Received 12 July 2013

Revised 30 September 2013

Available online 19 October 2013

\section{Keywords:}

Homonuclear $J$-coupling

Homonuclear decoupling

Transverse relaxation

Relaxation of antiphase terms

Spin dynamics

\begin{abstract}
A B S T R A C T
Homogeneous line-widths that arise from transverse relaxation tend to be masked by $B_{0}$ field inhomogeneity and by multiplets due to homonuclear $J$-couplings. Besides well-known spin-locking sequences that lead to signals that decay with a rate $R_{1} \rho$ without any modulations, alternative experiments allow one to determine the transverse relaxation rates $R_{2}$ in systems with scalar-coupled spins. We evaluate three recent strategies by experiment and simulation: (i) moderate-amplitude SITCOM-CPMG sequences (Dittmer and Bodenhausen, 2006 [2]), (ii) multiple-quantum filtered (MQF) sequences (Barrère et al., 2011 [4]) and (iii) PROJECT sequences (Aguilar et al., 2012 [5]). Experiments where the J-evolution is suppressed by spin-locking measure the pure relaxation rate $R_{2}\left(I_{x}\right)$ of an in-phase component. Experiments based on $J$ refocusing yield a mixture of in-phase rates $R_{2}\left(I_{x}\right)$ and antiphase rates $R_{2}\left(2 I_{y} S_{z}\right)$, where the latter are usually faster than the former. Moderate-amplitude SITCOM-CPMG and PROJECT methods can be applied to systems with many coupled spins, but applications of MQF sequences are limited to two-spin systems since modulations in larger systems can only partly be suppressed.
\end{abstract}

(c) 2013 Elsevier Inc. All rights reserved.
When speaking of "decoupling”, most NMR practitioners primarily think of collapsing multiplets stemming from J-couplings. In this article however, we shall focus on decoupling in time domain rather than in frequency domain. In other words, we will study methods that can 'smooth out' decays that are normally modulated by J-couplings. Indeed, the effects of couplings can be cancelled either in frequency or in time domain. In a heteronuclear system, e.g., comprising a proton coupled to a carbon-13 nucleus, a simple broadband radiofrequency $(r f)$ irradiation on one of the two nuclei is sufficient to achieve effective decoupling. This becomes challenging in the presence of homonuclear interactions. In such cases, the Larmor frequencies of the two nuclei can be very close and broadband $r f$ irradiation might perturb all spins, instead of affecting only one coupling partner. The same "homonuclear challenge" appears when attempting to measure the transverse relaxation rate $R_{2}=1 / T_{2}$ of such a spin system. If a spin echo is applied to a system of coupled protons, the echo envelope does not decay exponentially, but is modulated by homonuclear $J$-couplings. The first observation of such 'echo modulations' was made by Erwin

\footnotetext{
* Corresponding author: Address: Ecole polytechnique fédérale de Lausanne (EPFL), Institut des sciences et ingénierie chimiques, BCH 1529 (Batochime), $\mathrm{CH}-$ 1015 Lausanne, Switzerland.

E-mail address: geoffrey.bodenhausen@epfl.ch (G. Bodenhausen).

1 Present address: Laboratory of Physical Chemistry, ETH, 8093 Zürich, Switzerland.
}

Hahn while studying protons in ethanol shortly after the original discovery of spin echoes back in 1950 [1].

The spin echo is approaching an age where retirement is mandatory for many. Yet homonuclear decoupling and measurements of transverse relaxation rates in coupled spin systems remain an active field of research in NMR spectroscopy. In the last few years, several approaches were presented to tackle this challenge [2-5]. We recently reviewed the phenomenon of echo modulations $[6,7]$. In this paper, we would like to compare different pulse sequences and evaluate their suitability to determine reliable and robust transverse relaxation rates of coupled protons.

Molecules with coupled protons are common in chemistry. Although all measurements in this paper were conducted on proton resonances, they could be generalized to any other system with $J$-couplings between similar nuclei, e.g., molecules containing several ${ }^{19} \mathrm{~F}$ nuclei or that are isotopically enriched in carbon- 13 or nitrogen-15. The question "How can one measure transverse relaxation of protons?" is by no means new. In 1975, Freeman and Hill published an excellent review on this subject [8]. A first strategy in effect treats a homonuclear system as if it were a pseudo heteronuclear system. When the $r f$ amplitude $\omega_{1}$ of the $180^{\circ}$ pulse used to generate a spin echo is so low that only one coupling partner is affected, i.e., $\omega_{1} \ll \Omega_{S}$ (assuming two coupled spins $I$ and $S$ with the carrier on-resonance for spin $I$, far from the chemical shift $\Omega_{S}$ of spin $S$ ), only spin $I$ is flipped by the pulse while the off-resonance 
spin $S$ remains unaffected. In this case, the echo is not modulated, because the evolution under the homonuclear J-coupling is refocused in the same way as a chemical shift. The second strategy goes in an opposite direction by using strong, non-selective $180^{\circ}$ pulses in the multiple-echo scheme of Carr and Purcell [9], including the phase change suggested by Meiboom and Gill [10] $\left(90^{\circ}{ }_{y}-\left[\tau-180^{\circ}{ }_{x}-\tau\right]_{m}\right.$, where $m$ is an integer $)$, known today as the CPMG sequence. When the repetition rate $v_{\text {rep }}=1 /\left(\tau+\tau_{\pi}+\tau\right)$, where $\tau_{\pi}$ is the duration of the $180^{\circ}$ pulse, becomes high compared to the chemical shift of the off-resonance spin $S\left(v_{\text {rep }} \gg \Omega_{S} /(2 \pi)\right)$, echo modulations are quenched. This idea, first described by Gutowsky et al. [11] and by Allerhand [12], exploits the property that two magnetically equivalent nuclei lead to a singlet, since the $J$-coupling between them has no effect on their evolution, even when it does not vanish. A CPMG sequence with a sufficiently fast repetition rate leads to a similar effect as spin-locking with a continuous-wave (cw) rf irradiation [13]. In both cases, the two nuclei $I$ and $S$ are made temporarily equivalent so as to suppress the modulation due to $J_{I S}$. The corresponding decay time constant is usually called $R_{1} \rho$, for "longitudinal relaxation in the rotating frame", which is similar to $R_{2}$ in liquids but generally not identical. A classical text about relaxation in coupled spin systems is due to Vold and Vold [14].

One may ask at this point: why is it so important to measure transverse relaxation rates of protons exactly? Relaxation rates contain information about molecular structure and dynamics (in particular through the distance- and angle-dependence of dipoledipole couplings). The ratio between the longitudinal relaxation rate $R_{1}$ and the transverse relaxation rate $R_{2}$ allows one to determine the correlation time $\tau_{c}$ [15]. Hydrogen exchange rates can be quantified when both $R_{1}$ and $R_{2}$ relaxation rates are known $[16,17]$. For example, in NMR studies of proteins, the relaxation rates $R_{1}=1 / T_{1}$ and $R_{2}=1 / T_{2}$ of nitrogen-15 or carbon- 13 nuclei are often measured, while relaxation rates of protons are hardly ever discussed. The echo modulations due to $J$-couplings between protons are the main obstacle to such measurements. Yet transverse proton relaxation is commonly observed in rotating-frame Overhauser experiments (ROE), where the spins are locked and the $J$-modulation is therefore suppressed. Since protons are highly abundant, $T_{2}$ measurements of protons could simplify the access to information about molecular structure and dynamics. Furthermore, pulse sequences designed to measure unmodulated decays of proton echoes could play a role in magnetic resonance imaging (MRI) and in vivo spectroscopy, where $T_{2}$ 's of protons in virtually all molecules other than water enjoy increasing interest [18].

There are two fundamental approaches to inhibit or undo the evolution under chemical shifts: spin-locking or refocusing. Spinlocking prevents the chemical shift from evolving, while refocusing initially allows free evolution to occur, before changing sign to achieve a 'time reversal'. These two strategies can also be applied to $J$-couplings. We will now describe three recently proposed experiments that can be classified in terms of two limiting strategies: $J$-locking or $J$-refocusing of homonuclear couplings.

The first method, developed in our laboratory, employs a "moderate-amplitude SITCOM-CPMG sequence", and is based on an effective locking of the evolution under homonuclear $J$-couplings $[2,3]$. It is a conventional CPMG multiple-echo sequence that uses a moderate $r f$ amplitude $\omega_{1}$ for all $180^{\circ}$ echo pulses, where $\omega_{1}$ is of the same order of magnitude as the offset of the coupling partner(s) $\Omega_{s}$. The $r f$ carrier is set on the frequency of our spin of interest $I$. The off-resonance effect for spin(s) $S$ will introduce pulse errors, in analogy to the effects of tilted effective fields. This error is responsible for a conversion of antiphase coherence $2 I_{y} S_{z}$ into the multiple-quantum term $2 I_{y} S_{y}$. This effectively hinders the antiphase terms from building up and locks the in-phase coherence of the on-resonance spin $I_{x}$, an effect that was dubbed "Stabiliza- tion by Interconversion within a Triad of Coherences under Multiple Refocusing" (SITCOM).

On the other hand, a pulse sequence designed to refocus the homonuclear $J$-evolution was originally proposed by Takegoshi et al. [19] and by van Zijl et al. [20]. Again based on the CPMG sequence, a hard $90^{\circ}$ pulse with a phase orthogonal to the excitation pulse is inserted half-way between two $180^{\circ}$ pulses of a conventional CPMG sequence:

$90^{\circ}{ }_{y}-\left[\tau-180^{\circ}{ }_{x}-\tau-90^{\circ}{ }_{x}-\tau-180^{\circ}{ }_{x}-\tau\right]_{n}$

where $m=2 n$ is an integer.

In a two-spin system $I S$, the product operators before and after the central $90^{\circ}$ pulse are:

$$
\begin{aligned}
& \left(I_{x}+S_{x}\right) \cos \theta_{J}+\left(2 I_{y} S_{z}+2 I_{z} S_{y}\right) \sin \theta_{J} \stackrel{90^{\circ} x}{\rightarrow}\left(I_{x}+S_{x}\right) \cos \theta_{J} \\
& \quad-\left(2 I_{z} S_{y}+2 I_{y} S_{z}\right) \sin \theta_{J}
\end{aligned}
$$

where $\theta_{J}=\pi J 2 \tau$. For arbitrary values of $2 \tau$, the signal will be purely in-phase at the end of each block. This type of refocusing is well known for first-order quadrupolar interactions in 'quadrupolar echoes' [21] and for homonuclear dipolar interactions in solids in 'solid echoes' or 'dipolar echoes' [22]. All of these interactions have in common that they can be described by bilinear or quadratic spin operators [23]. For good reason, Takegoshi called this experiment "the perfect (PFCT) echo". This sequence can be run in the manner of Carr and Purcell's 'Method A' [9], by keeping the number of pulse cycles to $n=1$ and increasing the interval $\tau$ to monitor the decay of the echoes. Since all pulses are non-selective, the sequence is efficient over a broad bandwidth, in contrast to moderate-amplitude SITCOM-CPMG experiments. The drawback is that the $J$-refocusing only works perfectly for a two-spin system. When equivalent spins are present, as in $\mathrm{AX}_{2}$-systems, the oscillations are no longer completely suppressed. It was recently shown [5] that this limitation can be readily overcome by incrementing the number $n$ of pulse cycles and keeping the interval $\tau$ constant, in the manner of Carr and Purcell's 'Method B' [9], and by setting the $\tau$ interval so that $\theta_{J}=\pi J 2 \tau \ll 1$. This 'locks' the evolution due to $J$-couplings to further spins. This approach is less demanding than spin-locking over a range of chemical shifts, since $J<\Omega_{S} /(2 \pi)$ in weakly coupled spin systems. Morris and co-workers [5] called this approach PROJECT which stands for "Periodic Refocusing of $J$-Evolution by Coherence Transfer". Indeed, the exchange of the antiphase terms in Eq. (2) is achieved by a periodic symmetrical interconversion between $2 I_{y-}$ $S_{z}$ and $2 I_{z} S_{y}$ that amounts to a homonuclear coherence transfer.

Barrère et al. have described an experiment that bears similarities to the ideas of Takegoshi and van Zijl. The sum of the signals that can pass a zero-quantum filter (ZQF) on the one hand and a double-quantum filter (DQF) on the other is not modulated by $J$ couplings [4]. The sequence is similar to Eq. (1):

$90^{\circ}{ }_{y}-\left[\tau-180^{\circ}{ }_{x}-\tau-90^{\circ}{ }_{y} 90^{\circ}{ }_{\phi}-\tau-180^{\circ}{ }_{x}-\tau\right]$

There are now two $90^{\circ}$ pulses at the time of the first echo in the middle of the sequence. The first $90^{\circ}$ y pulse fails to refocus the $J$ coupling (due to its phase), but converts the antiphase terms $2 I_{y} S_{z}$ and $2 I_{z} S_{y}$ into a double-quantum coherence. The phase of the second $90^{\circ}{ }_{\phi}$ pulse and of the receiver are cycled to achieve multiplequantum filtration (MQF). We shall refer to this approach as the "MQF sequence". Like the PROJECT method, the MQF sequence is a broadband method, since it acts over a broad range of offsets. But since the MQF method is based on the summation of two experiments, one cannot increase the number of cycles $n$ by adding new blocks as in Eq. (1). This is why it remains limited to two-spin systems, since $\tau$ has to be varied to measure the echo decay. The simulated time evolution of the product operators for $(A)$ free 

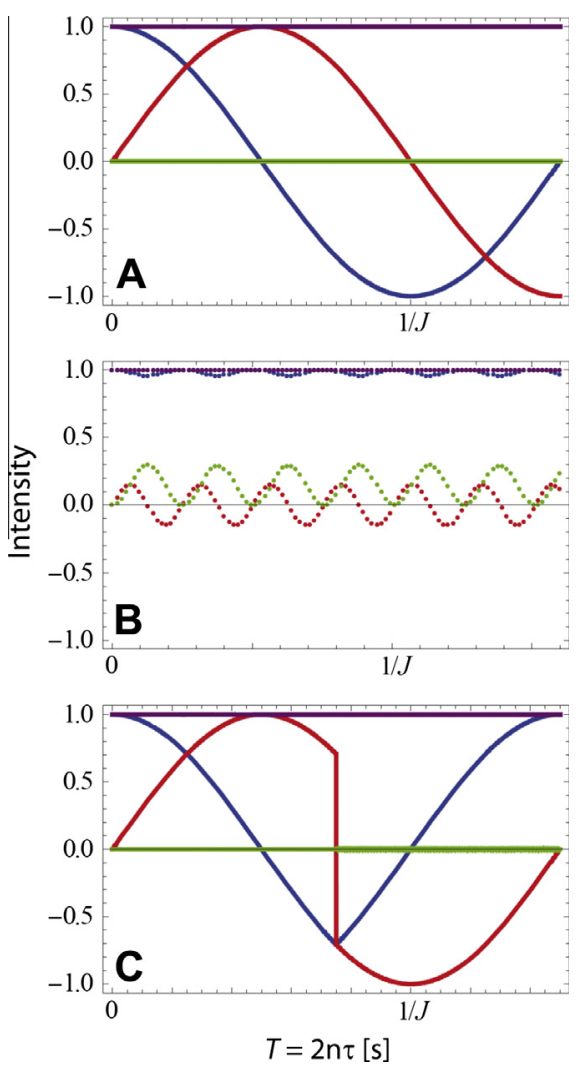

Fig. 1. Simulated time evolution of product operators for a $J$-coupled two-spin system IS. The spin of interest $I$ is on resonance $\Omega_{I} /(2 \pi)=0$, while spin $S$ has an offset of $\Omega_{S} /(2 \pi)=1 \mathrm{kHz}$, the coupling is $J=10 \mathrm{~Hz}$ and the initial state is $I_{x}+S_{x}$. The expectation values of the in-phase coherence $I_{x}$ are plotted in blue, the antiphase coherence $2 I_{y} S_{z}$ in red, the multiple-quantum coherence $2 I_{y} S_{y}$ in green and the norm of these three operators $N=\left(\left\langle I_{x}\right\rangle^{2}+\left\langle 2 I_{y} S_{z}\right\rangle^{2}+\left\langle 2 I_{y} S_{y}\right\rangle^{2}\right)^{1 / 2}$ in purple. Relaxation was not included. (A) Free evolution with a full interconversion of $I_{x}$ into $2 I_{y} S_{z}$. (B) SITCOM-CPMG sequence with 100 echoes separated by an inter-pulse delay of $2 \tau=1.5 \mathrm{~ms}$ and refocusing pulses with a moderate $r f$ amplitude of $\omega_{1} /(2 \pi)=5 \mathrm{kHz}$ $\left(\omega_{1} / \Omega_{S}=5\right)$. The in-phase coherence $I_{x}$ is effectively locked, because the build-up of $2 I_{y} S_{z}$ is hindered by the generation of the multiple-quantum coherence $2 I_{y} S_{y}$, resulting from the off-resonance effect on spin $S$ of the moderate-amplitude $r f$ pulses. (C) PFCT echo of Eq. (1) with $n=1$. After free evolution, the $90^{\circ}$ pulse in the middle of the sequence refocuses the in-phase coherence $I_{x}$, while inverting the antiphase coherence $2 I_{y} S_{z}$, but without reversing its sign (see Eq. (2)). In contrast to free evolution in (A), both (B) and (C) have $I_{x}=1$ and $2 I_{y} S_{z}=0$ at the end of their sequences. But while SITCOM-CPMG in (B) keeps $I_{x} \approx 1$ throughout the sequence, the term $I_{x}$ oscillates in the PFCT echoes (C). This leads to different effective relaxation rates in experiments for $(\mathrm{B})$ and $(\mathrm{C})$, because $2 I_{y} S_{z}$ usually decays faster than $I_{x}$. Simulations were performed with the SpinDynamica code developed by Malcolm H. Levitt, available at www.SpinDynamica.soton.ac.uk. (For interpretation of the references to color in this figure legend, the reader is referred to the web version of this article.).

evolution, (B) SITCOM-CPMG and (C) PFCT are shown in Fig. 1. A more detailed discussion of all these methods has been given elsewhere $[6,7]$.

All three methods lead to unmodulated echo decays, even in systems with homonuclear $J$-couplings. Nevertheless, these echoes do not all decay with the same rate. This is because the in-phase magnetization $I_{x}$ and the antiphase term $2 I_{y} S_{z}$ generally relax with different rates $R_{2}\left(I_{x}\right)$ and $R_{2}\left(2 I_{y} S_{z}\right)$ [14,24-29]. In practice, $R_{2}\left(2 I_{y} S_{z}\right)$ decays faster than $R_{2}\left(I_{x}\right)$ because of additional longitudinal relaxation pathways that affect spin $S$.

The free evolution in such a two-spin system can be described by two coupled differential equations in the basis $\left\{I_{x}, 2 I_{y} S_{z}\right\}$, where the solution can be expressed in the form of a Liouville equation $[29,30]$ :

$\sigma(t)=\exp \{L t\} \sigma(0)$ with

$L=\left(\begin{array}{cc}-R_{2}\left(I_{x}\right) & \pi J \\ -\pi J & -R_{2}\left(2 I_{y} S_{z}\right)\end{array}\right)$

The evolution of the density operator at the top of a selective echo can generally be written as

$\sigma(t)=(\exp \{L \tau\} \cdot P \cdot \exp \{L \tau\})^{m} \sigma(0)$

where

$P=\left(\begin{array}{cc}1 & 0 \\ 0 & -1\end{array}\right)$

is the operator of a selective $180^{\circ}$ rotation applied only on spin $I$, leaving $I_{x}$ unaffected but inverting the sign of $2 I_{y} S_{z}$ and $m$ is the number of echo cycles as defined above. Analytical solutions for the free evolution in Eq. (4) as well as at the time of the first echo $(m=1)$ after a selective $180^{\circ}$ x rotation on spin $I$ in Eq. (6) were given by Boulat and Bodenhausen [30]. Meersmann and Bodenhausen [31] interpreted this in terms of "relaxation-induced oscillations":

$$
\begin{aligned}
\sigma(2 \tau)= & I_{x}\left[\left(\frac{2 \pi J}{C}\right)^{2}-\frac{\Delta R_{2}}{C}\left(\frac{\Delta R_{2}}{C} \cos (C \tau)-\sin (C \tau)\right)\right] \times \exp \left\{-2 \tau \bar{R}_{2}\right\} \\
& -2 I_{y} S_{z} \frac{\Delta R_{2}}{C} \frac{2 \pi J}{C}[1-\cos (C \tau)] \times \exp \left\{-2 \tau \bar{R}_{2}\right\}
\end{aligned}
$$

With $\quad \bar{R}_{2}=\frac{1}{2}\left(R_{2}\left(I_{x}\right)+R_{2}\left(2 I_{y} S_{z}\right)\right), \quad \Delta R_{2}=\frac{1}{2}\left(R_{2}\left(2 I_{y} S_{z}\right)-R_{2}\left(I_{x}\right)\right)$, $C=\left[(2 \pi J)^{2}-\Delta R_{2}^{2}\right]^{1 / 2}$.

As shown in Fig. 1 of Ref. [31], $I_{x}$ oscillates around a mono-exponential decay with the average rate $\bar{R}_{2}$. If the parameter $\kappa=\left|R_{2} / 2 \pi J\right|$ is small, no oscillations are observed, since the $J$-coupling is large enough to average relaxation rates of in- and antiphase coherences. Such oscillations should not be confused with Hahn's echo modulations in a non-selective echo that can be explained without relaxation.

The time evolution under Takegoshi's perfect echo in Eq. (2) can be described in a similar way. Eqs. (5)-(7) can be applied for PFCT and PROJECT, since the antiphase terms are inverted by the $90^{\circ}{ }_{x}$ pulse in the middle of the sequence without affecting the in-phase coherences. To be consistent with our definition of the delay $\tau$ in Eq. (1), $\tau$ in Eqs. (5)-(8) should be replaced by $2 \tau$, since the perfect echo has the form of a double echo with four delays $\tau$ and $m$ should be replaced by $n$. Decays obtained by PFCT with $n=1$ may therefore show relaxation-induced modulations as described in Eq. (8). For PROJECT, Eq. (6) can be solved for a constant value of $\tau$, by incrementing $n$.

Strictly speaking, the basis $\left\{I_{x}, 2 I_{y} S_{z}\right\}$ is not sufficient to describe the time evolution under Eq. (2) for PFCT or PROJECT, since the central $90^{\circ}$ xulse not only inverts, but also exchanges the two antiphase terms $2 I_{y} S_{z}$ and $2 I_{z} S_{y}$. The corresponding Liouville equation needs to be extended to the basis $\left\{I_{x}, 2 I_{y} S_{z}, S_{x}, 2 I_{z} S_{y}\right\}$, but can still be written in the general form of Eq. (6) with

$L=\left(\begin{array}{cccc}-R_{2}\left(I_{x}\right) & \pi J & 0 & 0 \\ -\pi J & -R_{2}\left(2 I_{y} S_{z}\right) & 0 & 0 \\ 0 & 0 & -R_{2}\left(S_{x}\right) & \pi J \\ 0 & 0 & -\pi J & -R_{2}\left(2 I_{z} S_{y}\right)\end{array}\right)$

and

$P=\left(\begin{array}{cccc}1 & 0 & 0 & 0 \\ 0 & 0 & 0 & -1 \\ 0 & 0 & 1 & 0 \\ 0 & -1 & 0 & 0\end{array}\right)$ 
The solution will have a form similar to Eq. (8) with an oscillation around a mono-exponential decay, but the average rate $\bar{R}_{2}$ will contain the transverse relaxation rates of all four coherences involved in this basis. Likewise, the MQF sequence will decay with an average relaxation rate of several coherences.

Using only short values for $\tau$, PROJECT can suppress the modulations and partially hinder the interconversion, but it will still lead to an 'apparent transverse relaxation rate', i.e., a linear combination of $R_{2}\left(I_{x}\right), R_{2}\left(2 I_{y} S_{z}\right), R_{2}\left(S_{x}\right)$ and $R_{2}\left(2 I_{z} S_{y}\right)$. If $\tau$ is very short, the contribution of $R_{2}\left(2 I_{y} S_{z}\right)$ is small and the apparent transverse relaxation rate should be close to $R_{2}\left(I_{x}\right)$. Since $R_{2}\left(I_{x}\right)<R_{2}\left(2 I_{y} S_{z}\right)$ in practice, the 'contamination' of $R_{2}\left(I_{x}\right)$ by an admixture of $R_{2}\left(2 I_{y} S_{z}\right)$ leads one to overestimate the in-phase rate $R_{2}\left(I_{x}\right)$. On the other hand, moderate-amplitude CPMG benefits from the SITCOM effect and can yield a nearly 'pure' in-phase relaxation rate $R_{2}\left(I_{x}\right)$, provided $\tau \ll 1 / J$, as apparent from the inhibited build-up of the antiphase term $2 I_{y} S_{z}$ in Fig. 1(B). In some experiments, these details may be of secondary importance, particularly when the determination of an apparent $R_{2}^{\text {app }}$ is sufficient, but for a precise measurement of $R_{2}$, we cannot neglect these effects.

It is interesting to note that both pulse sequences described in Eqs. (1) and (3) appear to have been designed independently of "planar mixing" methods [32]. In contrast to "isotropic mixing" that is typically used for TOCSY experiments $[33,34]$ and employs an effective Hamiltonian $I \cdot S=I_{x} S_{x}+I_{y} S_{y}+I_{z} S_{z}$, planar mixing can use either a zero-quantum Hamiltonian $I_{x} S_{x}+I_{y} S_{y}$ or a doublequantum Hamiltonian $I_{x} S_{x}-I_{y} S_{y}$, neither of which are isotropic [32]. Planar mixing can be used to reduce the complexity of multiplets in homonuclear correlation experiments [32]. Planar mixing can also lead to spin waves [35]. Schulte-Herbrüggen et al. [32] started with a ZQ planar mixing sequence similar to Eq. (3) and simplified it to an expression similar to Eq. (1) of PFCT, by merging two $90^{\circ}$ pulses to a single $90^{\circ}$ pulse with a phase shift.

Therefore, while 'decoupling' with planar mixing sequences is based on anisotropic Hamiltonians, the SITCOM effect achieves 'decoupling' with an isotropic Hamiltonian. Note that decoupling with SITCOM breaks down for so-called 'recoupling conditions', when the pulse interval approximately matches the inverse of the chemical shift difference. At these conditions, the average Hamiltonian has the form $I_{z} S_{z}$, so that echo modulations reappear, as shown by Gopalakrishnan et al. [3]. Schulte-Herbrüggen et al. [32] explained that the zero- and double-quantum Hamiltonians and $I_{x} S_{x}+I_{y} S_{y}$ and $I_{x} S_{x}-I_{y} S_{y}$ have equivalent effects in two-spin systems, but that only isotropic Hamiltonians should be used for arbitrarily complex and strongly coupled spin system. These considerations do not include relaxation, but confirm that neither class of experiment should be affected by evolution under homonuclear $J$-couplings in a two-spin system.

For an experimental comparison of different methods to measure transverse relaxation rates, we consider simple $\mathrm{AX}$ and $\mathrm{AX}_{2}$ systems of coupled protons. All spectra were recorded at $500 \mathrm{MHz}\left(B_{0}=11.74 \mathrm{~T}\right)$ at $T=300 \mathrm{~K}$. The AX-system of the two protons in 2,3-dibromothiophene in $\mathrm{CD}_{2} \mathrm{Cl}_{2}$ has a chemical shift difference $\Delta \Omega /(2 \pi)=188 \mathrm{~Hz}$ and $J=5.8 \mathrm{~Hz}$. The $\mathrm{AX}_{2}$-system of 3,5-dichlorophenol in $\mathrm{CDCl}_{3}$ has a chemical shift difference $\Delta \Omega$ / $(2 \pi)=99.3 \mathrm{~Hz}$ and $J=1.75 \mathrm{~Hz}$. Echo decays were recorded in five different ways, using (i) as a reference, spin locking with a continuous-wave $r f$ irradiation to determine $R_{1}$, (ii) a moderate-amplitude CPMG sequence that benefits from the SITCOM effect, with a constant interval $\tau=1 \mathrm{~ms} \ll 1 / \mathrm{J}$ and an increasing number $n$ of refocusing pulses, in the manner of Carr and Purcell's method B, (iii) PROJECT echoes using the sequence of Eq. (1) with a constant interval $\tau=1 \mathrm{~ms} \ll 1 / \mathrm{J}$ and again an increasing number $n$ of refocusing cycles, (iv) PFCT echoes using the same sequence of Eq. (1) but with a single refocusing cycle $n=1$, while increasing the inter-pulse delays $\tau$ in the manner of Carr and Purcell's method
A, and, finally, (v) the MQF sequence with increasing inter-pulse delays $\tau$. The $r f$ carrier was set half-way between the two resonances for the spin-locking experiment (i), on-resonance for spin $I$ in the SITCOM-CPMG experiment (ii) and off-resonance for both spins $I$ and $S$ in experiments (iii), (iv) and (v).

Echo and spin-locking decays of the proton $\mathrm{H}^{3}$ in the AX-system of 2,3-dibromothiophene are shown in Fig. 2. The experimental conditions are summarized in Table 1 . As expected, none of the five decays suffer from significant echo modulations. The decay rates in Table 1 may be classified in three categories. The slowest decay rates are observed with the moderate-amplitude SITCOM-CPMG method (red circles), similar to the reference spin locking (blue squares). The corresponding exponential decays are nearly identical with $R_{2}=0.114 \mathrm{~s}^{-1}$ for SITCOM-CPMG and $R_{1 \rho}=0.119 \mathrm{~s}^{-1}$ for spin-locking. The PROJECT echoes (brown triangles), also lead to good exponential fits, albeit with a slightly faster decay $R_{2}=0.130 \mathrm{~s}^{-1}$. The two remaining experiments, the PFCT (green diamonds) and the MQF sequence (black stars) featured much faster decays that are contaminated by residual modulations. The oscillations could not be fitted by Eq. (8), since the frequency of the dips is smaller than $J$ and the amplitude of the modulations is larger than expected. The origin of this discrepancy could be due to strong coupling artefacts [36,37]. The (poor) fits led to $R_{2}=0.42 \pm 0.7 \mathrm{~s}^{-1}$ for the PFCT echoes and $R_{2}=0.39 \pm 0.3 \mathrm{~s}^{-1}$ for the MQF sequence.

The comparison of decays obtained with our moderate-amplitude SITCOM-CPMG sequences and spin-locking sequences that measure $R_{2}\left(I_{x}\right)$, respectively $R_{1}\left(I_{x}\right)$, shows that the decay rates measured with SITCOM-CPMG sequences are virtually equivalent to $R_{1} \rho$. This confirms that SITCOM-CPMG sequences with $\tau \ll 1 / J$ effectively lock the in-phase coherence $I_{x}$. PROJECT signals are free of modulations, but, as expected, do not relax with a "pure" rate $R_{2}\left(I_{x}\right)$, since a partial conversion into faster relaxing antiphase coherences cannot be inhibited.

The decays of the PFCT echoes and MQF sequences were 3-4 times faster than the others. Neither method involves stepping the number $n$ of refocusing cycles, since both increment the inter-pulse delays $\tau$ up to values $\tau \gg 1 / J$, where in- and antiphase coherences are freely interconverted. Knowing the in-phase rate $R_{2}\left(I_{x}\right)$ from spin-locking and SITCOM-CPMG measurements, we cannot determine $R_{2}\left(2 I_{y} S_{z}\right)$ since the transverse relaxation rates of spin $S R_{2}\left(S_{x}\right)$ and $R_{2}\left(2 I_{z} S_{y}\right)$ also contribute to the decays in PFCT and MQF sequences. A simple average of $R_{2}\left(I_{x}\right)$ and $R_{2}\left(2 I_{y} S_{z}\right)$ can be obtained by running a simple echo that is fully modulated by $\cos (\pi J t)$. If this assumption is valid, we can estimate the antiphase relaxation rate $R_{2}\left(2 I_{y} S_{z}\right)$ from the difference between the average rate $\bar{R}_{2}$ and the pure in-phase rate $R_{2}\left(I_{x}\right)$ :

$R_{2}\left(2 I_{y} S_{z}\right)=2 \bar{R}_{2}-R_{2}\left(I_{x}\right)$

To be consistent with the PFCT and MQF sequences, we measured a double echo with $m=2 n=2$ by incrementing $\tau$. The fully modulated decay (not shown) was fitted with the function $I(t)=\cos (\pi J t) \times \exp \left\{-\bar{R}_{2} t\right\}$ and $J=5.8 \mathrm{~Hz}$, leading to $\bar{R}_{2}=$ $0.45 \pm 0.7 \mathrm{~s}^{-1}$. Using Eq. (11), a surprisingly large rate $R_{2}\left(2 I_{y} S_{z}\right)-$ $\approx 0.8 \mathrm{~s}^{-1}$, about seven times faster than the pure in-phase rate was estimated, i.e., $R_{2}\left(2 I_{y} S_{z}\right) / R_{2}\left(I_{x}\right) \approx 7$. This ratio must be considered with caution, since for larger values $\tau$, diffusion may interfere, as seen for PFCT and MQF decays. By comparison, Meersmann and Bodenhausen [28] determined ratios of $1<R_{2}\left(2 I_{y} S_{z}\right) / R_{2}\left(I_{x}\right)<2$ for protons in Cyclosporin $\mathrm{A}$, albeit with different methods. By fitting the modulated decays of $z$-filtered echoes, Norwood found $R_{2}\left(2 I_{y-}\right.$ $\left.S_{z}\right) / R_{2}\left(I_{x}\right) \approx 26$ for a proton doublet in 5,7-dimethylcoumarin [27].

In the $\mathrm{AX}_{2}$-system of 3,5-dichlorophenol we shall focus on the triplet of the $\mathrm{H}^{4}$ proton. The echo decays shown in Fig. 3 correspond to the experimental conditions summarized in Table 2. The color coding of the different methods is the same as for the 


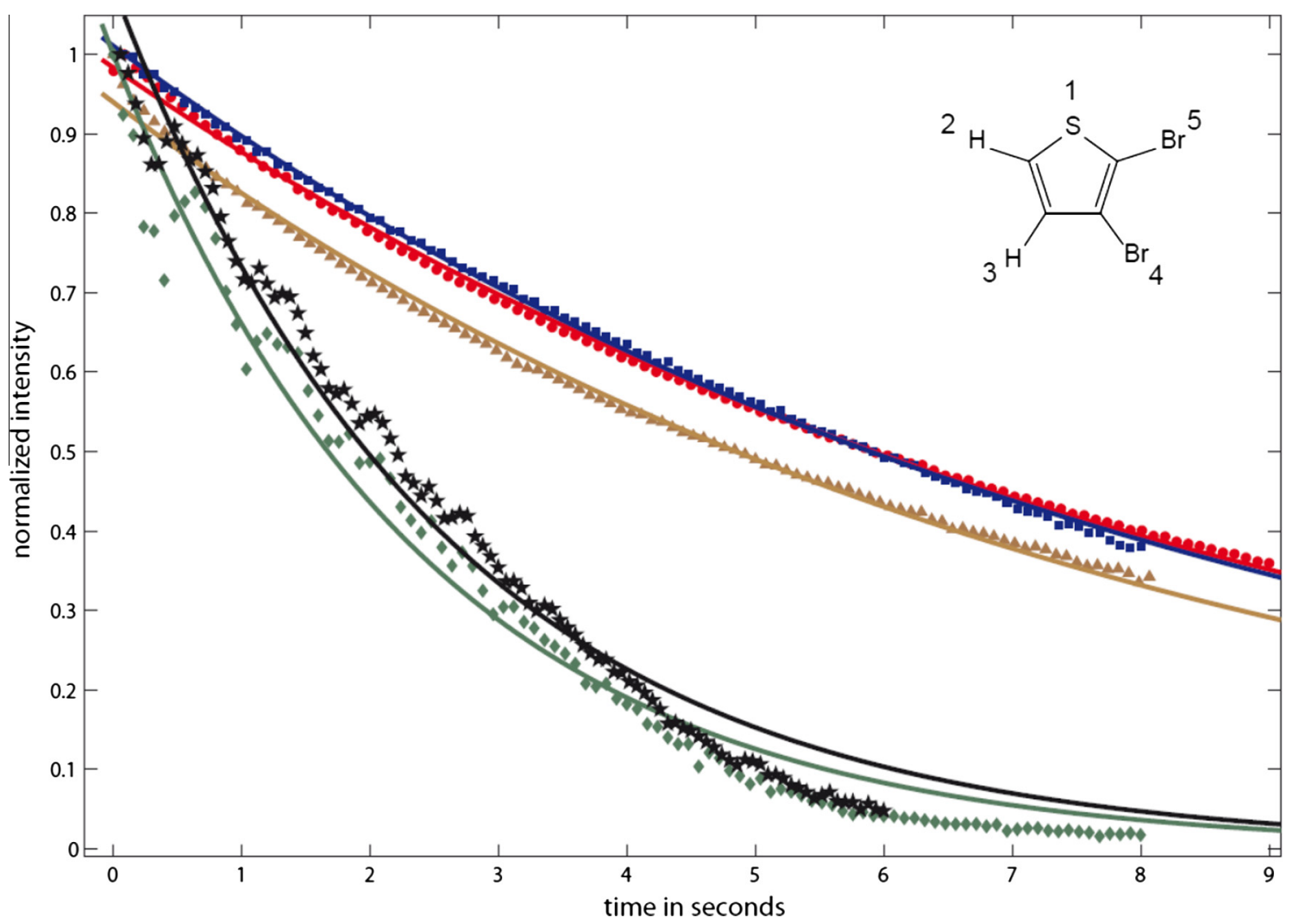

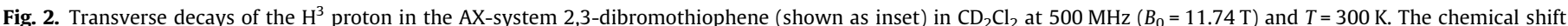

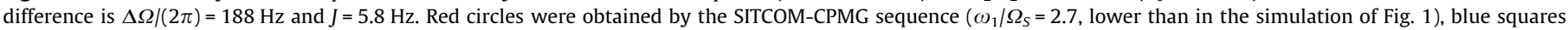

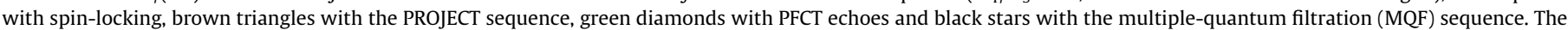

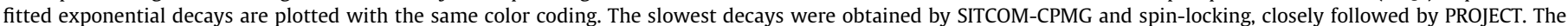

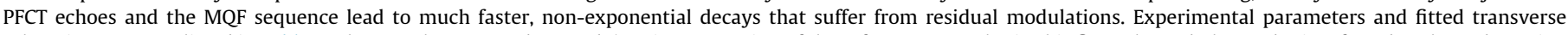

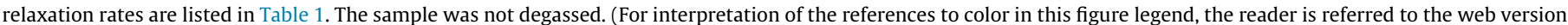
of this article.).

Table 1

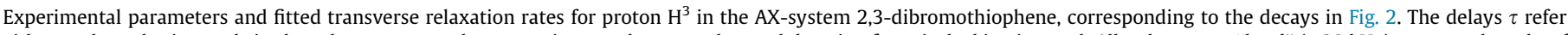

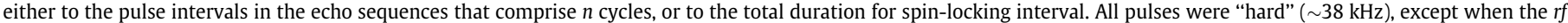
amplitude is mentioned in the table.

\begin{tabular}{|c|c|c|c|c|c|}
\hline Method & $R_{2}$ from fit & $\tau$ & $n$ & rf Carrier & Others \\
\hline (i) Spin locking & $0.119 \mathrm{~s}^{-1}$ (blue) & In steps of $80 \mathrm{~ms}$ to $8 \mathrm{~s}$ & - & Half-way between the two shifts & $\omega_{S L} /(2 \pi)=2 \mathrm{kHz}$ \\
\hline (ii) SITCOM-CPMG & $0.114 \mathrm{~s}^{-1}(\mathrm{red})$ & $1 \mathrm{~ms}$ & 1 , then in steps of $15-1500$ & On-resonance & $\omega_{1} /(2 \pi)=500 \mathrm{~Hz} \tau_{\pi}=1 \mathrm{~ms}$ \\
\hline (iii) PROJECT & $0.130 \mathrm{~s}^{-1}$ (brown) & $1 \mathrm{~ms}$ & 1 , then in steps of $20-2000$ & Off-resonance & - \\
\hline (iv) PFCT & $0.42 \pm 0.7 \mathrm{~s}^{-1}$ (green) & In steps of $20 \mathrm{~ms}$ to $2 \mathrm{~s}$ & 1 & Off-resonance & - \\
\hline (v) MQF & $0.39 \pm 0.3 \mathrm{~s}^{-1}$ (black) & In steps of $15 \mathrm{~ms}$ to $1.5 \mathrm{~s}$ & 1 & Off-resonance & 8-step phase cycle \\
\hline
\end{tabular}

AX-system in Fig. 2. While the order of the decay rates remains the same as for the AX-system, the decay rates measured by MQF (black stars) and PFCT echoes (green diamonds) are strongly modulated in the $\mathrm{AX}_{2}$-system. The modulations can even lead to negative intensities. As expected, the PFCT echo and MQF sequence fail to achieve proper decoupling effects in systems with more than two spins. Similar modulations in $\mathrm{AX}_{n}$-systems were discussed by van Zijl et al. [20]. Once again, this result proves the close relationship between MQF sequences and PFCT echoes. The short $\tau$ interval in the PROJECT sequence (brown triangles) leads to an oscillation-free decay, again as in Fig. 2, albeit with a slightly faster relaxation rate $R_{2}=0.208 \mathrm{~s}^{-1}$. Finally, the slowest rates were found using the moderate-amplitude SITCOM-CPMG $\left(R_{2}=0.149 \mathrm{~s}^{-1}\right)$ and the spin-locking sequence $\left(R_{1 \rho}=0.158 \mathrm{~s}^{-1}\right)$. It is interesting to note that the rates measured by SITCOM-CPMG were slightly smaller than $R_{1 \rho}$ in both spin systems investigated. This indicates that $R_{2}$ from SITCOM-CPMG are not significantly contaminated by small amounts of antiphase coherences. The rate $R_{1} \rho$ can depend on the amplitude of the $r f$ locking field $\omega_{S L}$. For both systems, we have chosen $\omega_{S L} \approx 10 \Delta \Omega$. This condition can only be fulfilled for small differences of chemical shifts, which often occur in proton spectra. It is more difficult to fulfill for other nuclei like carbon-13 which have a greater range of chemical shifts, so that heating by intense $r f$ fields cannot be excluded.

In contrast to the AX-system, it is difficult to estimate the antiphase relaxation rates $R_{2}\left(2 I_{y} S_{z}\right), R_{2}\left(2 I_{y} R_{z}\right)$ and $R_{2}\left(4 I_{y} S_{z} R_{z}\right)$ in the $\mathrm{AX}_{2}$-system of Fig. 3 (where spins $S$ and $R$ are magnetically equivalent). An echo decay fully modulated by $\cos ^{2}(\pi J t)$ would be the cleanest way to obtain an unbiased mixture of the in-phase rate $R_{2}\left(I_{x}\right)$ and all three antiphase rates $R_{2}\left(2 I_{y} S_{z}\right), R_{2}\left(2 I_{y} R_{z}\right)$ and $R_{2-}$ $\left(4 I_{y} S_{z} R_{z}\right)$. For a reliable calibration, an independent and direct measurement of the antiphase decay $R_{2}\left(4 I_{y} S_{z} R_{z}\right)$ would be desirable. 


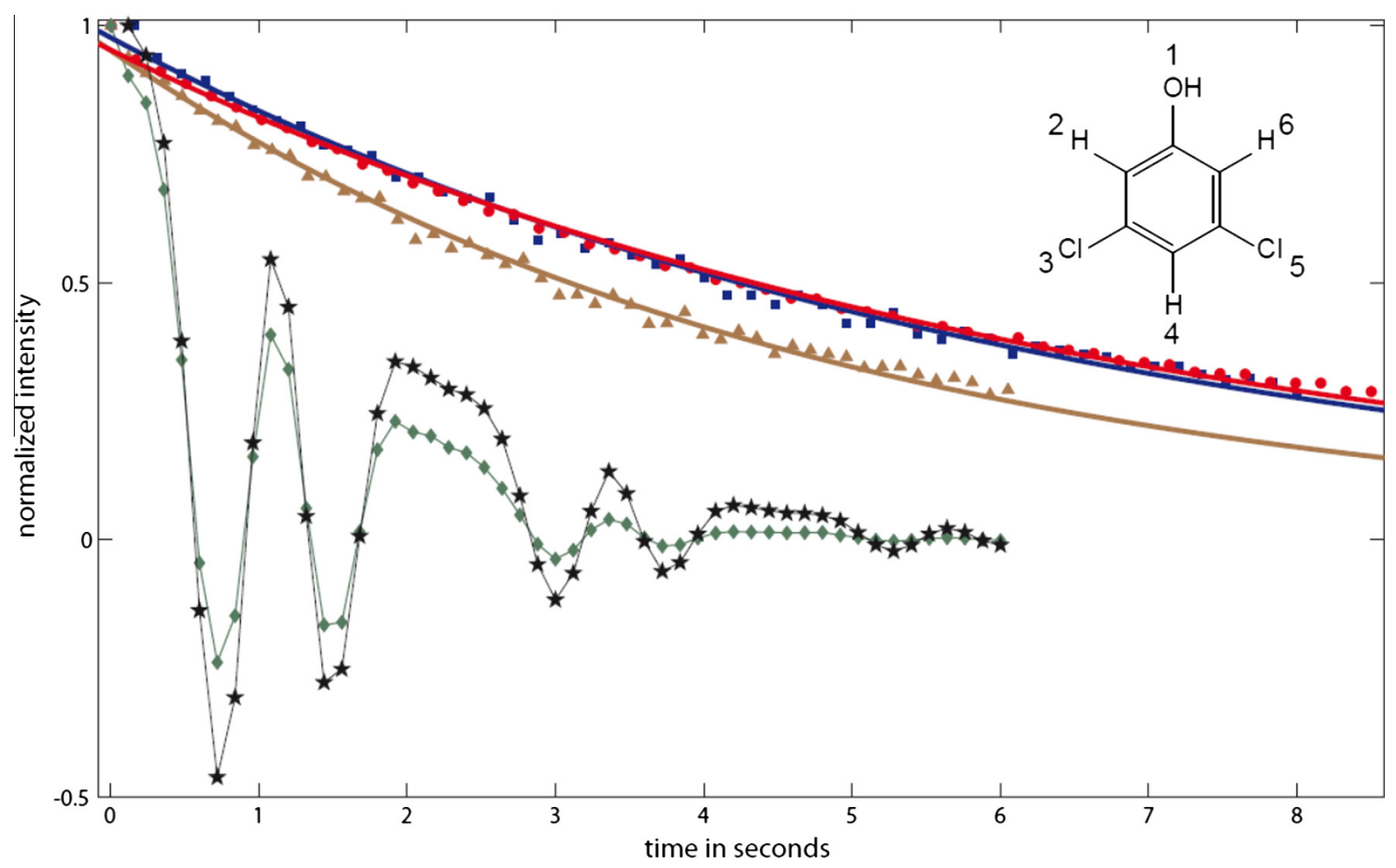

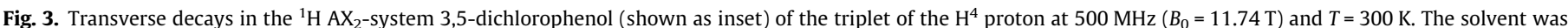

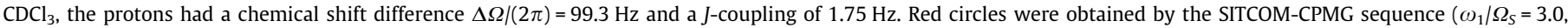

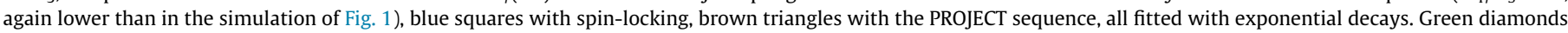

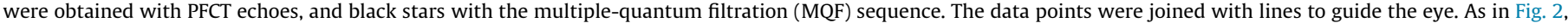

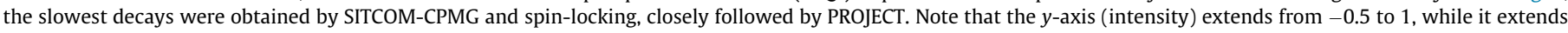
only from 0 to 1 in Fig. 2. Experimental parameters and the fitted relaxation rates are listed in Table 2.

Table 2

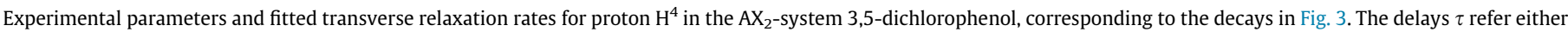

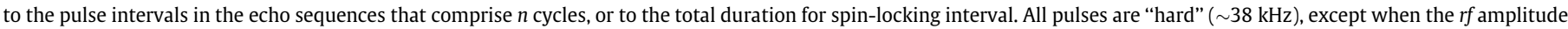
is mentioned in the table.

\begin{tabular}{|c|c|c|c|c|c|}
\hline Method & $R_{2}$ from fit & $\tau$ & $n$ & rf Carrier & Others \\
\hline (i) Spin locking & $0.158 \mathrm{~s}^{-1}$ (blue) & In steps of $160 \mathrm{~ms}$ to $8 \mathrm{~s}$ & - & Half-way between the two ${ }^{1} \mathrm{H}$ 's & $\omega_{S L} /(2 \pi)=1 \mathrm{kHz}$ \\
\hline (ii) SITCOM-CPMG & $0.149 \mathrm{~s}^{-1}(\mathrm{red})$ & $1 \mathrm{~ms}$ & 1 , then in steps of $30-1500$ & On-resonance & $\omega_{1} /(2 \pi)=300 \mathrm{~Hz} \tau_{\pi}=1 \mathrm{~ms}$ \\
\hline (iii) PROJECT & $0.208 \mathrm{~s}^{-1}$ (brown) & $1 \mathrm{~ms}$ & 1 , then in steps of $30-1500$ & Off-resonance & - \\
\hline (iv) PFCT & No fit (green) & In steps of $30 \mathrm{~ms}$ to $1.5 \mathrm{~s}$ & 1 & Off-resonance & - \\
\hline (v) MQF & No fit (black) & In steps of $30 \mathrm{~ms}$ to $1.5 \mathrm{~s}$ & 1 & Off-resonance & 8 -step phase cycle \\
\hline
\end{tabular}

As discussed above, the experiments illustrate that all sequences work off-resonance for both $\mathrm{AX}$ and $\mathrm{AX}_{2}$ spin systems, except for the moderate-amplitude SITCOM-CPMG. One may argue that the fastest method to obtain a reliable estimate of the rate $R_{2}$ in small molecules would be simply to measure $R_{1}$ by inversion-recovery. Indeed, if the correlation time $\tau_{c}$ is short enough, one can safely assume to be in the fast motion limit [38], so that $R_{2}=R_{1}$. However, this equality does not generally apply in coupled spin systems as demonstrated by Vold and Vold [39]. They showed in the same two-spin system of 2,3-dibromothiophene that the $R_{1}$ 's of the two protons were different while the $R_{1} \rho$ 's measured by spin-locking were identical. This effect was explained by an averaging of the rates of the two nuclei under strong $r f$ irradiation.

To conclude this experimental comparison, in-phase decays $R_{2}\left(I_{x}\right)$ in homonuclear systems are best determined either by spin-locking or by moderate-amplitude SITCOM-CPMG sequences. If the $r f$ amplitude $\omega_{S L}$ is sufficient, spin-locking has the advantage of being a broadband method, but it may cause heating. Moderateamplitude SITCOM-CPMG is a selective technique and may need some preliminary "hybrid" experiments or simulations to avoid recoupling conditions [3], but there is no risk of heating. As mentioned above, PROJECT offers an ideal solution for $T_{2}$-weighted experiments like DOSY: it yields clean exponential decays for all homonuclear spin systems in a broadband manner, although the generation of small amounts of antiphase coherences cannot be avoided. The PROJECT sequence was recently used to measure $T_{2}$ 's in proton spin systems [40] and also applied in CPMG-edited screening methods [41]. With PFCT echoes and MQF sequences, complete decoupling is limited to AX-systems and the decays reflect linear combinations of transverse relaxation rates of various coherences that depend on $\tau$. Furthermore, the MQF sequence needs an 8-step phase cycle [4]. Therefore, the use of these techniques to measure transverse relaxation in homonuclear coupled systems does not appear to be practical.

The measurement of the relaxation rate $R_{2}\left(2 I_{y} S_{z}\right)$ of an antiphase coherence is straightforward in heteronuclear systems: it is sufficient to apply a spin-locking $r f$ field to spin $I$, as proposed by Peng et al. in 1991 [25]. This strategy was adapted in 1995 by Meersmann for homonuclear systems [28] by selectively spinlocking the transverse components of antiphase terms associated 
with $H^{\alpha}$ and $H^{N}$ protons in Cyclosporin A (CsA), as well as an aromatic proton in a DNA dodecamer. To our knowledge, there have been no further attempts to measure antiphase decay rates of protons.

We adopted the scheme of Meersmann and applied a selective spin-locking field with $\omega_{S L} /(2 \pi)=30 \mathrm{~Hz}$ to the triplet of 3,5-dichlorophenol. The decay showed some modulations at the beginning and could not be properly fitted to a mono-exponential decay, in contrast to Meersmann's neat decays in Cyclosporin A (CsA). A biexponential fit allowed us to distinguish a fast rate of $1.29 \mathrm{~s}^{-1}$ and a slower one of $0.18 \mathrm{~s}^{-1}$. This may be explained by a much smaller chemical shift difference of $\Delta \Omega /(2 \pi)=99.3 \mathrm{~Hz}$ in our system compared to $\Delta \Omega /(2 \pi)=1.6 \mathrm{kHz}$ for an amide proton in CsA, both at $500 \mathrm{MHz}$. Since $\omega_{S L}$ must be large enough to suppress the $J$-evolution, but weak enough to avoid affecting the $S$ spin, we may have failed to find a middle road between these two conflicting demands. A locking field of $30 \mathrm{~Hz}$ applied to spin I may unwittingly affect spin $S$ so that $2 I_{y} S_{z}$ is partly converted into a multiplequantum coherence $2 I_{y} S_{x}$. On the other hand, if $\omega_{S L}$ is too weak, the $J$-evolution cannot be suppressed. A similar measurement in 2,3-dibromothiophene with $\omega_{S L} /(2 \pi)=20 \mathrm{~Hz}$ led to a bi-exponential fit with a fast rate of $3.4 \mathrm{~s}^{-1}$ and a slower one of $0.25 \mathrm{~s}^{-1}$.

Why is a stabilization of a homonuclear antiphase term $2 I_{y} S_{z}$ so difficult? In contrast to the in-phase term $I_{x}$, the antiphase product operator is bilinear and the phases are perpendicular. As seen above, there is always the risk of perturbing the $S$ spin in a homonuclear system when trying to stabilize the $I_{y}$ coherence. To meet this challenge, we might find some inspiration in recent strategies designed to preserve heteronuclear multiple-quantum coherences (MQC) to probe their relaxation dispersion $[42,43]$. Since all four coherences in the MQC subspace, i.e., $2 I_{x} S_{x}, 2 I_{x} S_{y}, 2 I_{y} S_{x}$, and $2 I_{y} S_{y}$ should be simultaneously preserved, a simple spin-locking field fails. The approach of Verde et al. is based on broadband heteronuclear decoupling schemes such as MLEV and WALTZ $[44,45]$. Could a composite-pulse sequence exist that would preserve homonuclear antiphase terms? Further simulations and experiments will be needed. Measuring the relaxation rates of antiphase terms in homonuclear systems remains a challenging task.

The fastest way to determine transverse decays of a pure inphase coherence $I_{x}$ in proton systems remains spin-locking, which is broadband and works for any kind of spin system. The moderateamplitude SITCOM-CPMG method leads to the same $R_{2}\left(I_{x}\right)$, but every resonance has to be measured in turn, which can become quite time consuming [46-48]. Some preliminary experiments may be needed to ensure that recoupling conditions can be avoided. The advantage over spin-locking is that SITCOM-CPMG will not lead to any significant heating. This may be less relevant in the case of protons with a narrow distribution of chemical shifts, but it can become important in systems that are isotopically enriched in carbon-13 which are known to have extensive homonuclear couplings $[49,50]$.

\section{Acknowledgments}

We thank Dr. Bikash Baishya, Dr. Diego Carnevale and Dr. Sami Jannin for helpful discussions, Martial Rey for technical support and the Swiss National Science Foundation, the Commission for Technology and Innovation, the Ecole Polytechnique Fédérale de Lausanne and the CNRS for support.

\section{References}

[1] E.L. Hahn, Spin echoes, Phys. Rev. 80 (1950) 580-594.

[2] J. Dittmer, G. Bodenhausen, Quenching echo modulations in NMR spectroscopy, ChemPhysChem 7 (2006) 831-836.

[3] K. Gopalakrishnan, N. Aeby, G. Bodenhausen, Quenching and recoupling of echo modulations in NMR spectroscopy, ChemPhysChem 8 (2007) 1791-1802.
[4] C. Barrère, P. Thureau, A. Thévand, S. Viel, A convenient method for the measurements of transverse relaxation rates in homonuclear scalar coupled spin systems, Chem. Commun. 47 (2011) 9209-9211.

[5] J.A. Aguilar, M. Nilsson, G. Bodenhausen, G.A. Morris, Spin echo NMR spectra without J modulation, Chem. Commun. 48 (2012) 811-813.

[6] T.F. Segawa, Decoupling of Magnetic Resonance Signals in Time and Frequency Domain, EPFL, PhD Thesis, 2013, doi: 10.5075/epfl-thesis-5688.

[7] T.F. Segawa, G. Bodenhausen, Modulations of spin echoes in liquids, in: eMagRes, vol. 2, John Wiley \& Sons Ltd., 2013, pp. 245-252.

[8] R. Freeman, H.D.W. Hill, in: L.M. Jackman, R.D. Adams (Eds.), Dynamic Nuclear Magnetic Resonance Spectroscopy, Academic Press, New York, 1975.

[9] H.Y. Carr, E.M. Purcell, Effects of diffusion on free precession in nuclear magnetic resonance experiments, Phys. Rev. 94 (1954) 630-638.

[10] S. Meiboom, D. Gill, Modified spin-echo method for measuring nuclear relaxation times, Rev. Sci. Instrum. 29 (1958) 688-691.

[11] H.S. Gutowsky, R.L. Vold, E.J. Wells, Theory of chemical exchange effects in magnetic resonance, J. Chem. Phys. 43 (1965) 4107-4125.

[12] A. Allerhand, Analysis of Carr-Purcell spin-echo NMR experiments on multiple-spin systems. I. Effect of homonuclear coupling, J. Chem. Phys. 44 (1966) 1-9.

[13] I. Solomon, Etude de la relaxation en résonance magnétique par la méthode de "précession forcée transitoire", C. R. Acad. Sci. Paris 248 (1959) 92-94.

[14] R.L. Vold, R.R. Vold, Nuclear magnetic-relaxation in coupled spin systems, Prog. Nucl. Magn. Reson. Spectrosc. 12 (1978) 79-133.

[15] J. Kowalewski, L. Mäler, Nuclear Spin Relaxation in Liquids: Theory, Experiments, and Applications, Taylor \& Francis, 2006.

[16] S. Meiboom, Z. Luz, D. Gill, Proton relaxation in water, J. Chem. Phys. 27 (1957) 1411-1412.

[17] F. Kateb, P. Pelupessy, G. Bodenhausen, Measuring fast hydrogen exchange rates by NMR spectroscopy, J. Magn. Reson. 184 (2007) 108-113.

[18] S. Michaeli, M. Garwood, X.H. Zhu, L. DelaBarre, P. Andersen, G. Adriany, H. Merkle, K. Ugurbil, W. Chen, Proton T-2 relaxation study of water, Nacetylaspartate, and creatine in human brain using Hahn and Carr-Purcell spin echoes at 4 T and 7 T, Magn. Reson. Med. 47 (2002) 629-633.

[19] K. Takegoshi, K. Ogura, K. Hikichi, A perfect spin-echo in a weakly homonuclear J-coupled 2 spin-1/2 system, J. Magn. Reson. 84 (1989) 611-615.

[20] P.C.M. van Zijl, C.T.W. Moonen, M. von Kienlin, Homonuclear-J refocusing in echo spectroscopy, J. Magn. Reson. 89 (1990) 28-40.

[21] M. Bloom, E.L. Hahn, B. Herzog, Free magnetic induction in nuclear quadrupole resonance, Phys. Rev. 97 (1955) 1699-1709.

[22] J.G. Powles, P. Mansfield, Double-pulse nuclear-resonance transients in solids, Phys. Lett. 2 (1962) 58-59.

[23] O.W. Sorensen, G.W. Eich, M.H. Levitt, G. Bodenhausen, R.R. Ernst, Product operator-formalism for the description of NMR pulse experiments, Prog. Nucl. Magn. Reson. Spectrosc. 16 (1983) 163-192.

[24] M. Goldman, Interference effects in the relaxation of a pair of unlike spin-1/2 nuclei, J. Magn. Reson. 60 (1984) 437-452.

[25] J.W. Peng, V. Thanabal, G. Wagner, Improved accuracy of heteronuclear transverse relaxation-time measurements in macromolecules - elimination of antiphase contributions, J. Magn. Reson. 95 (1991) 421-427.

[26] J.W. Peng, G. Wagner, Mapping of spectral density-functions using heteronuclear NMR relaxation measurements, J. Magn. Reson. 98 (1992) 308-332.

[27] T.J. Norwood, Measurement of the scalar coupling and transverse relaxationtimes of doublets, J. Magn. Reson. Ser. A 101 (1993) 109-112.

[28] T. Meersmann, G. Bodenhausen, Transverse relaxation in proton NMR separate measurement of decay-rates of in-phase and antiphase coherences, J. Magn. Reson. Ser. A 115 (1995) 277-282.

[29] J. Cavanagh, W.J. Fairbrother, A.G. Palmer, M. Rance, N.J. Skelton, Protein NMR Spectroscopy Principles and Practice, second ed., Elsevier Academic Press, Amsterdam, 2007.

[30] B. Boulat, G. Bodenhausen, Measurement of proton relaxation rates in proteins, J. Biomol. NMR 3 (1993) 335-348.

[31] T. Meersmann, G. Bodenhausen, Relaxation-induced oscillations of spin-echo envelopes, Chem. Phys. Lett. 257 (1996) 374-380.

[32] T. Schulte-Herbrüggen, Z.L. Madi, O.W. Sorensen, R.R. Ernst, Reduction of multiplet complexity in COSY-type NMR-spectra - the bilinear and planar COSY experiments, Mol. Phys. 72 (1991) 847-871.

[33] L. Braunschweiler, R.R. Ernst, Coherence transfer by isotropic mixing application to proton correlation spectroscopy, J. Magn. Reson. 53 (1983) 521-528.

[34] R.P. Hicks, J.K. Young, D. Moskau, Magnetization transfer via isotropic mixing: an introduction to the HOHAHA experiment, Concepts Magn. Reson. 6 (1994) $115-130$.

[35] Z.L. Madi, B. Brutscher, T. Schulte-Herbrüggen, R. Brüschweiler, R.R. Ernst, Time-resolved observation of spin waves in a linear chain of nuclear spins, Chem. Phys. Lett. 268 (1997) 300-305.

[36] G. Bodenhausen, R. Freeman, G.A. Morris, D.L. Turner, NMR spectra of some simple spin systems studied by two-dimensional Fourier transformation of spin echoes, J. Magn. Reson. 31 (1978) 75-95.

[37] M.J. Thrippleton, R.A.E. Edden, J. Keeler, Suppression of strong coupling artefacts in J-spectra, J. Magn. Reson. 174 (2005) 97-109.

[38] J. Keeler, Understanding NMR Spectroscopy, second ed., Wiley, 2011.

[39] R.L. Vold, R.R. Vold, Averaging of transverse relaxation-times of scalar coupled nuclei under spin lock conditions, J. Chem. Phys. 61 (1974) 25252529. 
[40] L. Skjærven, L. Codutti, A. Angelini, M. Grimaldi, D. Latek, P. Monecke, M.K. Dreyer, T. Carlomagno, Accounting for conformational variability in proteinligand docking with NMR-guided rescoring, J. Am. Chem. Soc. 135 (2013) 5819-5827.

[41] I.K.H. Leung M. Demetriades, A.P. Hardy, C. Lejeune, T.J. Smart, A. Szöllössi, A Kawamura, C.J. Schofield, T.D.W. Claridge, Reporter ligand NMR screening method for 2-oxoglutarate oxygenase inhibitors, J. Med. Chem. 56 (2013) 547555.

[42] M. Verde, S. Ulzega, F. Ferrage, G. Bodenhausen, Preservation of heteronuclear multiple-quantum coherences in NMR by double-resonance irradiation, J. Chem. Phys. 130 (2009) 074506.

[43] S. Ulzega, M. Verde, F. Ferrage, G. Bodenhausen, Heteronuclear double resonance in nuclear magnetic resonance spectroscopy: relaxation of multiple-quantum coherences, J. Chem. Phys. 131 (2009) 224503.

[44] R. Freeman, Spin Choreography Basic Steps in High Resolution NMR, Spektrum, 1997.
[45] A.J. Shaka, J. Keeler, Broadband spin decoupling in isotropic liquids, Prog. Nucl. Magn. Reson. Spectrosc. 19 (1987) 47-129.

[46] B. Baishya, T.F. Segawa, G. Bodenhausen, Apparent transverse relaxation rates in systems with scalar-coupled protons, J. Am. Chem. Soc. 131 (2009) 17538 17539.

[47] T.F. Segawa, B. Baishya, G. Bodenhausen, Transverse relaxation of scalarcoupled protons, ChemPhysChem 11 (2010) 3343-3354.

48] T.F. Segawa, B. Baishya, G. Bodenhausen, Transverse relaxation of scalar coupled protons in magnetic resonance of non-deuterated proteins, Appl Magn. Reson. 42 (2012) 353-361.

[49] N. Aeby, G. Bodenhausen, Determination of transverse relaxation rates of individual spins while quenching echo modulations due to homonuclear scalar couplings, Chem. Phys. Lett. 463 (2008) 418-421.

[50] T.F. Segawa, N. Aeby, G. Bodenhausen, Apparent transverse relaxation rates in systems with coupled carbon-13 spins, Phys. Chem. Chem. Phys. 12 (2010) 9772-9776. 